\title{
Possible Role of Gamma Ray Bursts on Life Extinction in the Universe
}

\author{
Tsvi Piran ${ }^{1, *}$ and Raul Jimenez $z^{2,3, \dagger}$ \\ ${ }^{1}$ Racah Institute of Physics, The Hebrew University, Jerusalem 91904, Israel \\ ${ }^{2}$ ICREA and ICC, University of Barcelona, Marti i Franques 1, Barcelona 08028, Spain \\ ${ }^{3}$ Institute for Applied Computational Science, Harvard University, Massachusetts 02138, USA
}

(Received 11 September 2014; published 5 December 2014)

\begin{abstract}
As a copious source of gamma rays, a nearby galactic gamma ray burst (GRB) can be a threat to life. Using recent determinations of the rate of GRBs, their luminosity function, and properties of their host galaxies, we estimate the probability that a life-threatening (lethal) GRB would take place. Amongst the different kinds of GRBs, long ones are most dangerous. There is a very good chance (but no certainty) that at least one lethal GRB took place during the past 5 gigayears close enough to Earth as to significantly damage life. There is a 50\% chance that such a lethal GRB took place during the last $500 \times 10^{6}$ years, causing one of the major mass extinction events. Assuming that a similar level of radiation would be lethal to life on other exoplanets hosting life, we explore the potential effects of GRBs to life elsewhere in the Galaxy and the Universe. We find that the probability of a lethal GRB is much larger in the inner Milky Way (95\% within a radius of $4 \mathrm{kpc}$ from the galactic center), making it inhospitable to life. Only at the outskirts of the Milky Way, at more than $10 \mathrm{kpc}$ from the galactic center, does this probability drop below $50 \%$. When considering the Universe as a whole, the safest environments for life (similar to the one on Earth) are the lowest density regions in the outskirts of large galaxies, and life can exist in only $\approx 10 \%$ of galaxies. Remarkably, a cosmological constant is essential for such systems to exist. Furthermore, because of both the higher GRB rate and galaxies being smaller, life as it exists on Earth could not take place at $z>0.5$. Early life forms must have been much more resilient to radiation.
\end{abstract}

DOI: 10.1103/PhysRevLett.113.231102

PACS numbers: 98.70.Rz, 89.60.Gg, 98.52.-b

Introduction.-Gamma ray bursts (GRBs), short and intense bursts of $\gamma$ rays, are the brightest explosions known. The copious flux of $\gamma$-ray photons with energies above $100 \mathrm{keV}$ from a galactic GRB could destroy the ozone layer, making them potentially damaging to life on Earth. This has led to the suggestion [1-9] that events of massive life extinction were caused by galactic GRBs. (See [10] for an earlier discussion of nearby supernovae as the cause of life extinction.) This issue depends, of course, on the rate of galactic GRBs in the Earth's neighborhood. Once it was realized that long GRBs are preferentially located at lowmetallicity environments, it was claimed [11] that nearby Galactic GRB are rare and GRBs are unlikely to play any role in life extinction on Earth (see however, Ref. [12], which claims that metallicity won't protect life on Earth from GRBs). Given the recent significant progress in quantifying the main ingredients that determine whether GRBs have any effect on Earth - their rate, luminosity function, and dependence on metallicity - it is therefore timely to reassess this issue, extending the discussion to GRB effects on life in the entire Milky Way and the whole Universe.

GRBs are traditionally divided into two groups, depending upon their duration: long ( $>2$ s) GRBs (LGRBs) and short $(<2 \mathrm{~s})$ GRBs (sGRBs). This division follows to a large extent the origin of these events. (We note in passing that some GRBs that are shorter than $2 \mathrm{~s}$ do arise from collapsing massive stars [13]. However, this is unimportant for this Letter.) LGRBs are associated with the death of massive stars (see, e.g., Ref. [14] for a review), while sGRBs have a different origin, most likely compact binary mergers [15]. Recently, it was realized that there is a third group characterized by low luminosity $\left(L \approx 10^{46-48} \mathrm{erg} \mathrm{s}^{-1}\right.$ ) and denoted $l l$ GRBs. These events are also associated with the death of massive stars, but they originate from a different physical mechanism [16]. A fourth type of a related explosion-giant flares (SGR) - might also be relevant. Such a flare took place in the Milky Way on Dec 27, 2004, releasing $\approx 4 \times 10^{46} \mathrm{ergs} \mathrm{[17].} \mathrm{This} \mathrm{flare,} \mathrm{which} \mathrm{was}$ sufficiently powerful to disturb the Earth's ionosphere was seen as a brief change in the ionization levels in the lowest regions of the Earth's ionosphere (the $D$ layer), is the only known object outside the solar system to have a direct clear impact on Earth. In fact, this type of disturbance was first seen from a GRB830801 in 1983 [18]. Giant SGR flares are a different phenomenon than GRBs, but, as their rates could be as high as once every 30 years in the Galaxy, we will explore their possible role as well. Solar flares are another potential life-threatening source, as they are stronger than previously thought [19-21].

Wanderman and Piran [22] have recently reconstructed, in a model independent way, the rate of LGRBs as a function of redshift and their luminosity function. One of their most interesting findings is that the LGRB rate is not reproduced by the star formation rate of the global galaxy population. This discrepancy is statistically highly significant, particularly at low $(<3)$ redshifts, which is relevant here. This is, at 
first, surprising, as there is ample evidence that long duration GRBs originate from the collapse of very massive stars, and one would expect the LGRB to follow the star formation rate (SFR). Jimenez and Piran [23] have shown that the LGRB rate and the galaxy derived SFR agree for a special class of galaxies: low mass (stellar mass $<10^{10} \mathrm{M}$ ) and low metallicity ( $\lesssim 1 / 10$ solar). This is, of course, done in a statistical sense and does not exclude the possibility that few outliers to this trend exist. But it is clear that the LGRB host population is a special subclass of the general galaxy population. These results are in agreement with earlier observations that indicate that LGRBs take place in dwarf [24], low-metallicity [25] galaxies. They are also consistent with direct observations of LGRB host metallicities (e.g., Refs. [26-28]) and with the findings of Fruchter et al. [29], who have shown that the local SFR in the vicinity of LGRBs is much higher than expected if they simply follow the general SFR of the host galaxy (see also Ref. [30]).

sGRBs have very different host environments and they clearly arise from different progenitors (see, e.g., Refs. [31,32] for reviews). They are significantly weaker than LGRBs, and as such are observed at much shorter distances than LGRB. sGRBs are believed to originate in compact binary mergers [15], but a direct proof for this is still lacking. As sGRBs are weaker, fewer GRBs have been observed than LGRBs. However, their current overall rate is about 5 times larger than the rate of LGRBs. In the following we use a recent determination of the sGRB's global rate and luminosity function by Wanderman and Piran [33].

llGRBs are significantly weaker than both LGRBs and sGRBs, with energies of $10^{47-49} \mathrm{ergs}$, and also smoother and softer. Like LGRBs they are associated with the death of massive stars, but they arise due to a different physical mechanism [16]. While less than half a dozen $l l$ GRBs have been observed so far, they are more numerous than both LGRBs and sGRBs [34]. Because of their low luminosities, they are observed only up to relatively short (but still cosmological) distances.

We use the very recent determination of GRB rates and luminosity function to estimate the flux of Galactic GRBs on Earth and compare it with the flux needed to destroy the ozone layer. Given that LGRBs are the most powerful bursts - and hence the most dangerous - and also given their dependence on metallicity, we begin with an exposition of the Milky Way metallicity distribution. We continue estimating the life-threatening effect of LGRBs, turning later, using the same formalism, to sGRBs, $l l \mathrm{GRBs}$, and giant SGR flares. We conclude by summarizing the results and their implication regarding life extinction on Earth. We also explore the implications to life extinction on exoplanets elsewhere in the Milky Way and in the whole Universe.

The Milky Way metallicity distribution.-LGRB rate estimates derive the expected rates of LGRBs per unit volume per unit time. When translating this volumetric rate to event rate per galaxy and more specifically to the rate within the Milky Way, one has to consider the type of galaxies in which the events take place. Our earlier analysis [23] shows that LGRB hosts are dwarf low metallicity galaxies that are very different from the Milky Way. There are outliers, and some LGRBs have been found in higher metallicity galaxies [26,27].

Reference [35] and references therein determine the ages and metallicities of stars in the Milky Way disk. Figure 1 depicts the percentage distribution of stars in the Milky Way for ages $<1$ gigayear (Gyr) (the solid black line) and stars older than $1 \mathrm{Gyr}$ but younger than $5 \mathrm{Gyr}$ (the solid orange line). Stars that are older than the Sun and that therefore trace the chemical conditions of the star forming gas at earlier epochs are not relevant for the question of life destruction on Earth. In the same plot we also show (the solid green line) the percentage distribution of LGRB hosts derived from Ref. [23], using the mass metallicity relation from Ref. [36]. Note that because of the metallicity bias for the LGRB host galaxies, there is very little overlap with the distribution of stars in the Milky Way disk. In fact they overlap only at the $10 \%$ level.

Also shown in Fig. 1 are the distributions of LGRB hosts with direct metallicity determinations (the dashed blue lines) as compiled in Ref. [26] and those of GRB host metallicities derived from damped Ly- $\alpha$ (DLA) measurements (red line), as reported in Ref. [28]. The percentage of overlap of direct host metallicities with those of stars in the Milky Way is $10 \%$. We conclude that the metallicity bias will reduce the probability for LGRB within the last 5 Gyrs in the Milky Way by a percentage between 5\% (from the metallicity determination in Ref. [23]) and 10\% [from direct metallicity determinations (e.g., Refs. [26-28])] resulting in a reduction factor between 10 and 20 as compared to the volumetric rate

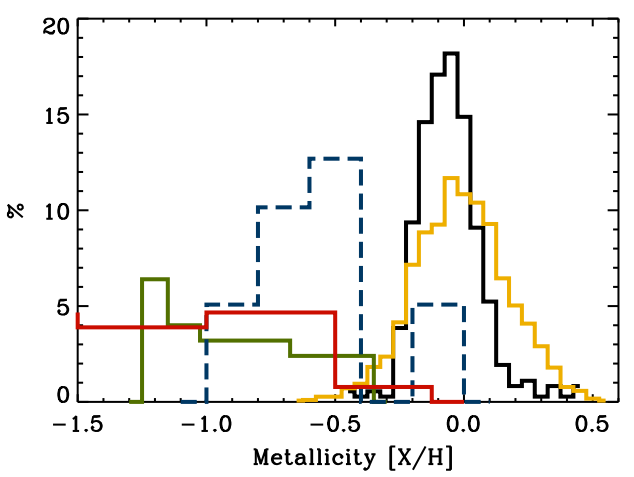

FIG. 1 (color online). The percentage of stars as a function of metallicity in the Milky Way disk with ages $1<t / \mathrm{Gyr}<5$ (the solid orange line) and with ages $<1$ Gyr (the solid black line), as obtained by Casagrande et al. [35]. The distribution of LGRB metallicity, as obtained by Jimenez and Piran [23], by matching the RGB global rate to the global star formation rate of galaxies (the solid green line) and that from direct metallicity determinations of LGRBs (the dashed line) [26] and Cucchiara et al. [28] from DLA systems (solid red line). The overlap between the LGRB and Milky Way star distributions is only at the few $\%$ level. 
TABLE I. Parameters of the LGRB and sGRB luminosity functions from Wanderman and Piran [22,33]. Note that the upper and lower limits are not well determined, but this is unimportant for our estimates here.

\begin{tabular}{lcccccc}
\hline \hline & $n_{0} \mathrm{Gpc}^{-3} \mathrm{yr}^{-1}$ & $\hat{\alpha}$ & $\hat{\beta}$ & $L^{*} \mathrm{ergs} \mathrm{s}^{-1}$ & $L_{\min } \mathrm{ergs} \mathrm{s}^{-1}$ & $L_{\max } \mathrm{ergs} \mathrm{s}^{-1}$ \\
\hline LGRB & $0.15_{-0.8}^{+0.7}$ & $1.2_{-0.1}^{+0.2}$ & $2.4_{-0.6}^{+0.3}$ & $10^{52.5 \pm 0.2}$ & $10^{49}$ & $10^{54}$ \\
sGRB & $0.04_{-0.019}^{+0.023}$ & $1.9 \pm 0.12$ & $3.0_{-0.8}^{+1}$ & $10^{52.3 \pm 0.2}$ & $5 \times 10^{49}$ & $10^{53}$ \\
\hline \hline
\end{tabular}

of LGRBs. In what follows we will assume a conservative $10 \%$ value for a metallicity bias for LGRB above solar.

Life threatening GRBs in the Milky Way.-Following Wanderman and Piran $[22,33]$ we write the current $(z=0)$ luminosity function as

$$
\phi(L)=n_{0}\left\{\begin{array}{ll}
\left(L / L^{*}\right)^{-\hat{\alpha}} & L_{\min }<L<L^{*} \\
\left(L / L^{*}\right)^{-\hat{\beta}} & L^{*}<L<L_{\max }
\end{array} .\right.
$$

The parameters of the luminosity functions are given in Table I and the functions are shown in Fig. 2. [The luminosity function defined here, $\phi(L)$, is per $d L / L *$. As such, it differs from that given in Refs. [22,33], which is per $d \log _{10}(L)$. The power law indices are marked by ${ }^{\wedge}$ to denote this difference. Clearly, $\hat{\alpha}=\alpha+1$ and $\hat{\beta}=\beta+1$.] This luminosity and rate are the isotropic equivalent (namely, disregarding the poorly constrained beaming), which are the quantities needed for our estimates here. In the following we need the total energy (see also Ref. [37]) and not the peak luminosity. A good but rough estimate is obtained by assuming a typical duration of $20 \mathrm{~s}(1 \mathrm{~s})$ for LGRBs (sGRBs). Multiplying by the average ( half) of the peak flux we obtain $E_{\mathrm{LGRB}}=10 L$ and $E_{\mathrm{sGRB}}=0.5 \mathrm{~L}$. In what follows we adopt the cosmological volume occupied by a Milky Way-type galaxy as $10^{-7} \mathrm{Gpc}^{3}$

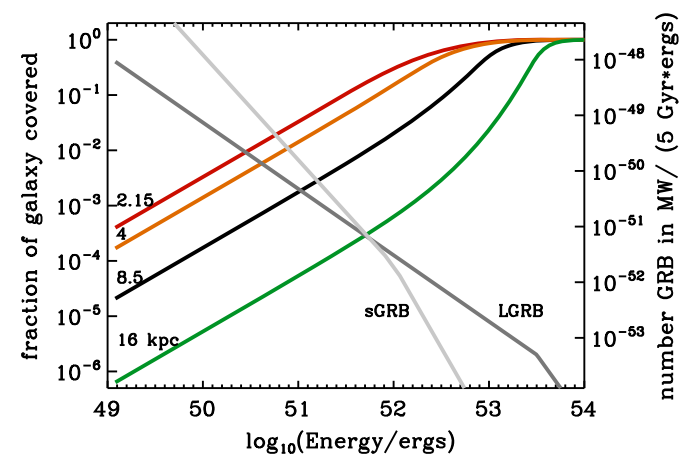

FIG. 2 (color online). (Left $y$ axis) The mass fraction of the galaxy from which the fluence on a planet will exceed $100 \mathrm{~kJ} / \mathrm{m}^{2}$ for a given explosion energy (the $x$ axis). The colored curves correspond to different locations of the life-harboring exoplanet $(2.15,4,8.5$, and $16 \mathrm{kpc}$ from the Galactic center). We have adopted for the MW an exponential disc with a scale length of $2.15 \mathrm{kpc}$. The right $y$ axis provides (for the gray curves) the number of GRBs per erg in the MW in the past 5 Gyr. For a given energy, the product of the corresponding colored and gray curves gives the number of damaging GRBs to life per energy interval. [see, e.g., Panter et al. [38] (Fig. 3), where we use $6 \times 10^{10} M$ as the stellar mass of the Milky Way [39]].

Assuming that GRBs follow the stellar distribution, they are distributed in the exponential disk of the Milky Way with a radial density profile given by $\rho \propto \exp \left(-r / r_{d}\right)$, with $r_{d}=2.15 \pm 0.14 \mathrm{kpc}$ (a number that, surprisingly, has only been accurately determined recently [40]). Using this density profile we calculate $p[d, R]$, the fraction of the Galaxy within a distance $d$ from a position $R$ (see Fig. 2). The expected number of GRBs, with a fluence exceeding $\mathcal{F}$ at a location at distance $R$ from the Galactic center, is:

$$
\langle N\rangle=\int_{L_{\min }}^{L_{\max }} \phi(L) p[d(E, \mathcal{F}), R] d L .
$$

To estimate the effect of a GRB on life on Earth we need to know what the dangerous radiation doses are. Ruderman [10], who considered at the time the effect of a nearby supernova on Earth, realized that the most damaging effect would be the depletion of the Earth's protective ozone layer
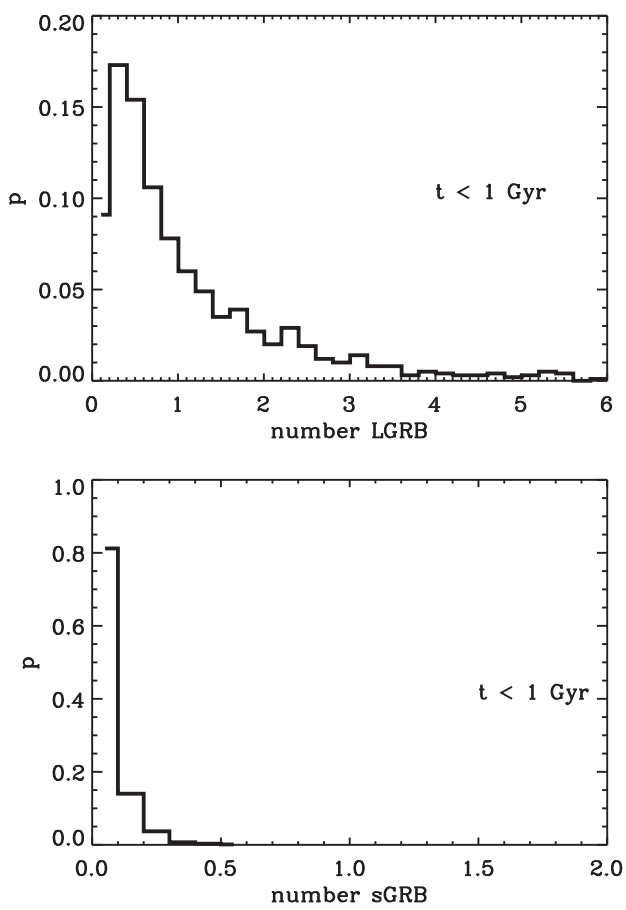

FIG. 3. The probability distribution function, $p$, of the average number of lethal LGRBs (top panel) and sGRBs (bottom panel) that irradiated Earth in the past Gyr with enough flux to cause severe life extinction $\left(100 \mathrm{~kJ} / \mathrm{m}^{2}\right)$. For LGRBs we show the case where we applied a $10 \%$ metallicity bias. 
for a period of months. This would happen via a formation of stratospheric nitric oxide that destroys the ozone. The ozone depletion would lead to enhancement of UVB solar radiation that, in turn, would be harmful to life. Note that the UVB fluence on the surface of the ocean will destroy surface marine life (as described in detail in Ref. [7]), including plankton, which will deprive (marine) life of their main nutrient. In 1995, after it was realized that GRBs are cosmological and their rate was estimated, Thorsett [1] applied these ideas to Galactic GRBs. A decade later Thomas et al. [6,7] carried out the most extensive, to date, calculation of the effects of gamma ray flux on the Earth's atmosphere. They found that a fluence of $10 \mathrm{~kJ} / \mathrm{m}^{2}$ will cause a depletion of $68 \%$ of the ozone layer in a time scale of a month. Fluences of $100 \mathrm{~kJ} / \mathrm{m}^{2}$ and $1000 \mathrm{~kJ} / \mathrm{m}^{2}$ will cause depletions of $91 \%$ and $98 \%$, respectively. One has to realize that these are average quantities. The exact amount of depletion depends on the direction of the GRB as well as on the season when the GRB takes place, and it may vary from one latitude to another. Following Thomas et al. [6,7] we estimate that a fluence of $10 \mathrm{~kJ} / \mathrm{m}^{2}$ will cause some damage to life, while $1000 \mathrm{~kJ} / \mathrm{m}^{2}$ will wipe out nearly the whole atmosphere, causing a catastrophic life extinction event; we consider $\mathcal{F}=100 \mathrm{~kJ} / \mathrm{m}^{2}$ as our canonical life threatening fluence. We do not consider here other sources of damage, such as the possibility that cosmic rays (CRs) are associated with the GRBs and that those could lead to enhanced radioactivity in the atmosphere $[2,3]$. The mean free path for deflection in the galactic magnetic field for a $100 \mathrm{GeV}$ proton is $1 \mathrm{kpc}$. So, the lowest part of the CR spectrum which contains the largest number of CRs will be deflected and will not reach Earth if the event is more than $1 \mathrm{kpc}$ away. This also means that while we will eventually get CR flux from GRBs that do not point towards Earth, a single event will always be less powerful (because of the deflecting away of CRs) so that their effect will be weakened and, depending on their spectrum, significantly weakened.

Integrating over the luminosity functions in Eq. (2), we estimate $\langle N\rangle$ for both long and short GRBs. These values are listed in Table II. To estimate the significance of these numbers, taking into account the errors in the luminosity function, burst duration, and the Milky Way disk scale length, we carry out a Monte Carlo simulation of 1000 realizations for both long and short GRBs. We calculate the distribution of $\langle N\rangle$ and the overall probability of more than one life-threatening GRB taking place within the last $5 \mathrm{Gyr}$, $1 \mathrm{Gyr}$, and $500 \times 10^{6}$ years.

An inspection of Fig. 2 reveals that maximal danger arises from $\sim L^{*}$ bursts. Lower luminosity bursts are more abundant, but their covering fraction of the Galaxy is too small. Higher luminosity bursts can destroy life in a large fraction of the Galaxy, but they are extremely rare. From the point of view of computational certainty, these results are reassuring, as the confidence in our determination of the rate of events around $L^{*}$ is good. This is also important from another point
TABLE II. Probability, in \%, of at least one GRB having occurred in the past time $t$ with enough flux to produce significant life extinction. For LGRB we show the probability without parentheses when there is a $10 \%$ metallicity bias, in parentheses when there is none. We consider three cases of the GRB fluence on Earth $\left(10,100\right.$, and $\left.1000 \mathrm{~kJ} / \mathrm{m}^{2}\right)$.

\begin{tabular}{lccc}
\hline \hline & $t<5 \mathrm{Gyr}$ & $t<1 \mathrm{Gyr}$ & $t<0.5 \mathrm{Gyr}$ \\
\hline $10 \mathrm{~kJ} / \mathrm{m}^{2}$ & & & \\
$\mathrm{LGRBs}$ & $99.8(99.95)$ & $98.7(99.90)$ & $95(99.80)$ \\
$\mathrm{sGRBs}$ & 80 & 37 & 22 \\
$l l \mathrm{GRBs}$ & $<1$ & $<1$ & $<1$ \\
$100 \mathrm{~kJ} / \mathrm{m}^{2}$ & & & \\
$\mathrm{LGRBs}$ & $90(99.8)$ & $60(96)$ & $50(90)$ \\
$\mathrm{sGRBs}$ & 14 & 3 & 2 \\
$l l \mathrm{GRBs}$ & $<1$ & $<1$ & $<1$ \\
$1000 \mathrm{~kJ} / \mathrm{m}^{2}$ & & $7(40)$ & $4(25)$ \\
$\mathrm{LGRBs}$ & $25(80)$ & $2 \times 10^{-3}$ & $10^{-3}$ \\
$\mathrm{sGRBs}$ & $10^{-2}$ & 0 & 0 \\
$l l \mathrm{GRBs}$ & 0 & & \\
\hline \hline
\end{tabular}

of view. Spatially, GRBs are concentrated within regions of the highest SFR $[29,30]$. The dominance of strong GRBs whose radius of influence is a few kpc implies that we can ignore this spatial inhomogeneity, and the approximation that the distribution of LGRBs follows the distribution of matter in the galaxy holds.

We find that the probability of a LGRB, in the past $5 \mathrm{Gyr}$, with a fluence of $100 \mathrm{~kJ} / \mathrm{m}^{2}$ on Earth to be higher than $90 \%$, and in the last $0.5 \mathrm{Gyr}$ this probability is $50 \%$. It is somewhat surprising that this result (a $50 \%$ chance of a biospherically important event in a half Gyr) is so similar to the original calculation in Thorsett [1]. At a lower fluence, $10 \mathrm{~kJ} / \mathrm{m}^{2}$, these probabilities are higher than $99.8 \%(95 \%)$ for $5 \mathrm{Gyr}(0.5 \mathrm{Gyr})$, and thus are nearly certain. However, the chances of a truly catastrophic event with a fluence of $1000 \mathrm{~kJ} / \mathrm{m}^{2}$ are at most $25 \%$, thus making it unlikely. These probabilities are, of course, much larger (see Table II) if we ignore the suppression of GRBs in the Milky Way due to large metallicity.

sGRBs are weaker and, as such, even though their rate is larger than the rate of LGRBs (and particularly so in the Milky Way because of the metallicity bias), their life-threatening effect is negligible (as can be seen from Table II). As $l l$ GRBs are even weaker, their effect is completely negligible. For completeness we mention that a giant SGR flare would have to be within $\sim 1$ to $2 \mathrm{pc}$ from Earth to produce a $100 \mathrm{~kJ} / \mathrm{m}^{2}$ fluence. This is comparable to the distance between stars in the solar neighborhood. Consequently, giant SGR flares are unlikely to cause any significant damage to life.

GRBs and life in the Galaxy.-We turn now to exploring the possible threat caused by GRBs to life elsewhere in the Milky Way, turning to the whole Universe in the next section. Clearly, to do so one must assume the lethal radiation dose that will be threatening to life elsewhere. 
While life can take numerous other forms and could be much more resilient to radiation elsewhere than on Earth, we here make the conservative assumption that life is rather similar to that on Earth. This common assumption is the basis for searches of Earth-like exoplanets as places that harbor life. Under this assumption, we explore what the likelihood is that a nearby GRB will result in a dose of 100 as well as 10 and $1000 \mathrm{~kJ} / \mathrm{m}^{2}$ in various regions of the Milky Way.

The stellar density is significantly larger towards the center of the Galaxy; hence, the threat to life on most exoplanets that reside in this region is much larger. Figure 4 depicts the probability of having one life-threatening event within the last $1 \mathrm{Gyr}$ as a function of the distance $r$ of an exoplanet from the Galactic center. (We use $1 \mathrm{Gyr}$ as a round number to estimate life extinctions that could have caused a massive extinction that terminated life and thus made it unlikely that we find signs of life today.) Also shown is the fraction of the stellar population of the Milky Way within this radius. A lethal GRB of $100 \mathrm{~kJ} / \mathrm{m}^{2}$ would be more than $95 \%$ likely up to a distance of $2 \mathrm{kpc}$ from the Galactic center, in which $25 \%$ of the Milky Way (MW) stars reside. When considering $\mathcal{F}=10$ and $1000 \mathrm{~kJ} / \mathrm{m}^{2}$, we find 12 and $0.5 \mathrm{kpc}$, respectively. In agreement with the specific estimates for Earth, events around the solar distance from the Galactic could be significant but rare, and only at a distance $>10 \mathrm{kpc}$ does the threat from GRBs become small. Therefore, life can be preserved with certainty only in the outskirts of our Galaxy. In total $90 \%, 40 \%$, and $5 \%$ of the exoplanets in the MW would be exposed to a fluence of 10,100 , and $1000 \mathrm{~kJ} / \mathrm{m} 2$ from GRBs within a period of $1 \mathrm{Gyr}$.

Finally, given the LGRBs' luminosity function, there are practically no lethal events with a distance larger than $30 \mathrm{kpc}$. This implies that nearby small satellite galaxies with a large SFR, like the Large Magellanic Cloud, are too far away to influence life in the Milky Way. The fact that the local group is such a low density region-containing

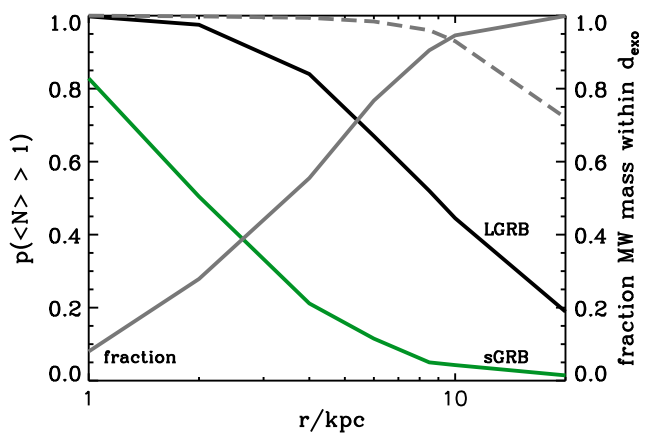

FIG. 4 (color online). The probability, $P(\langle N\rangle)$, of having on average more than one lethal GRB in the past Gyr for an exoplanet at a distance $r$ from the center of the Milky Way. The gray line shows the fraction of mass in the Milky encompassed within a radius $r$. The dashed line is for LGRB, assuming no metallicity correction. only two large galaxies (Andromeda and the Milky Way) and with the nearest cluster of galaxies, Virgo, at $16 \mathrm{Mpc}$, i.e., much farther away than the typical intergalactic distance of $1 \mathrm{Mpc}$ - seems to provide the required environment to preserve life on Earth. There is no threat from nearby extragalactic bursts.

GRBs and life in the Universe.-Before concluding, we turn now to consider the conditions elsewhere in the Universe. We have already mentioned that the local neighborhood of the Milky Way has a lower density of star forming dwarf galaxies, making the Milky Way a more friendly neighborhood for life. We can take our calculation one step further and compute the effective volume in the Universe protected from GRB explosions for life proliferation. This happens for galaxies that produce enough metals so that their metallicity is at least $1 / 3$ solar and their stellar disks are larger than $4 \mathrm{kpc}$. Using the mass-metallicity relation in Fig. 6 of Panter et al. [36], such galaxies must have stellar masses larger than $10^{10} \mathrm{M}$. This corresponds to a comoving abundance of $10^{-3}$ galaxies per $\mathrm{Mpc}^{3}$ (see Fig. 3 of Panter et al. [38]). This is a factor 10 less than the abundance of the most common galaxies. Galaxies friendly to harboring and preserving life will preferably inhabit low density regions in voids and filaments of the cosmic web.

Turning to earlier epochs we may wonder whether life could have existed in the earlier Universe. We recall that the age of the Universe at $z=1$ is about $6 \mathrm{Gyr}$, so, in principle, there was enough time for life to evolve even before this redshift; here we note that the LGRB rate was significantly larger in the past, making the GRB threat much more significant. Furthermore, galaxies at high $z$ are smaller than the current ones by a factor of 2-4 in radius and as such have less room for isolated safe regions like the outskirts of the Milky Way. We conclude that it is impossible to harbor life at $z>0.5$, as LGRBs will always be sufficiently near life-harboring planets and thus will cause life extinctions. It seems that the survival of life as we know it on Earth is only a recent phenomenon in the history of the Universe caused by the growth of large galaxies. Life-forms that might have existed earlier or that exist today in other regions of the Universe that are much more susceptible to significant GRB bombardment must be much more resilient to radiation than life on Earth. Of course, we do not know whether destruction of a large fraction of life and lifeforms on a given planet is good or bad for the long-term evolution of higher life-forms on that planet, only that it would be highly damaging for the existing higher lifeforms - including humans on our own planet right nowand that is what this study, in essence, concerns.

Conclusions. - We have used the latest determination of GRB rates and luminosities to estimate the likelihood of them being the source of life extinction on Earth. Using also the latest determinations of metallicity of stars in the Milky Way and those of LGRB hosts, we have concluded that the likelihood of a GRB producing life extinction on 
Earth is high. Taking the same lethal dose for extraterrestrial life as for life on Earth, we have found that GRBsand, in particular, LGRBs - are life threatening in a large part of the Milky Way as well as in many other locations in the Universe. The safest environments to preserve life are the outskirts of large galaxies in low density regions (so that these galaxies do not have "dangerous" low metallicity dwarf satellites). It is interesting to point out that a cosmological constant of about the same order of magnitude as the present value is essential for the Universe to grow large galaxies and also preserve low density regions at late times $z<0.5$; the expansion history of a $\lambda$ cold dark matter universe is modified in such a way that it provides enough time at high $z$ for large underdensities and galaxies to grow large. It is also worth mentioning that the damaging nature of GRBs could help explain Fermi's paradox. We will investigate both of these questions in detail in a forthcoming publication.

T. P. thanks the Institut Lagrange de Paris for their hospitality while this work was being completed. This research was supported by the ERC grant GRBs, by the ISF I-Core center of excellence, and by an Israel-China grant. R. J. thanks the Royal Society and the ICIC at Imperial College for their financial support and hospitality while this work was being completed. We thank Chris Flynn and Luca Casagrande for the discussions on the age-metallicity relation of stars in the Milky Way and the anonymous referees for their constructive and useful comments.

*tsvi.piran@huji.ac.il

†raul.jimenez@icc.ub.edu

[1] S. E. Thorsett, Astrophys. J. 444, L53 (1995).

[2] A. Dar, A. Laor, and N. J. Shaviv, Phys. Rev. Lett. 80, 5813 (1998).

[3] A. Dar and A. De Rujula, arXiv:astro-ph/0110162.

[4] J. Scalo and J. C. Wheeler, Astrophys. J. 566, 723 (2002).

[5] A. L. Melott, B. S. Lieberman, C. M. Laird, L. D. Martin, M. V. Medvedev, B. C. Thomas, J. K. Cannizzo, N. Gehrels, and C. H. Jackman, Int. J. Astrobiol. 3, 55 (2004).

[6] B. C. Thomas, C. H. Jackman, A. L. Melott, C. M. Laird, R. S. Stolarski, N. Gehrels, J. K. Cannizzo, and D. P. Hogan, Astrophys. J. Lett. 622, L153 (2005).

[7] B. C. Thomas, A. L. Melott, C. H. Jackman, C. M. Laird, M. V. Medvedev, R. S. Stolarski, N. Gehrels, J. K. Cannizzo, D. P. Hogan, and L. M. Ejzak, Astrophys. J. 634, 509 (2005).

[8] A. L. Melott and B.C. Thomas, Paleobiology 35, 311 (2009).

[9] P. A. Karam, Health Phys. 82, 491 (2002).

[10] M. A. Ruderman, Science 184, 1079 (1974).
[11] K. Z. Stanek et al., Acta Astronaut. 56, 333 (2006).

[12] A. L. Melott, arXiv:astro-ph/0604440.

[13] O. Bromberg, E. Nakar, T. Piran, and R. Sari, Astrophys. J. 764, 179 (2013).

[14] S. E. Woosley and J.S. Bloom, Annu. Rev. Astron. Astrophys. 44, 507 (2006).

[15] D. Eichler, M. Livio, T. Piran, and D. N. Schramm, Nature (London) 340, 126 (1989).

[16] O. Bromberg, E. Nakar, and T. Piran, Astrophys. J. Lett. 739, L55 (2011).

[17] D. M. Palmer, S. Barthelmy, N. Gehrels et al., Nature (London) 434, 1107 (2005).

[18] G. J. Fishman and U. S. Inan, Nature (London) 331, 418 (1988).

[19] A. L. Melott and B.C. Thomas, Astrobiology 11, 343 (2011).

[20] A. L. Melott and B. C. Thomas, Nature (London) 491, E1 (2012).

[21] I. G. Usoskin, B. Kromer, F. Ludlow, J. Beer, M. Friedrich, G. A. Kovaltsov, S. K. Solanki, and L. Wacker, Astron. Astrophys. 552, L3 (2013).

[22] D. Wanderman and T. Piran, Mon. Not. R. Astron. Soc. 406, 1944 (2010).

[23] R. Jimenez and T. Piran, Astrophys. J. 773, 126 (2013).

[24] P. Natarajan, J. S. Bloom, S. Sigurdsson, R. A. Johnson, N. R. Tanvir, P. J. Groot, T. J. Galama, J. Van Paradijs, and C. Kouveliotou, New Astron. 2, 471 (1997).

[25] J. P. U. Fynbo, P. Jakobsson, P. Möller et al., Astron. Astrophys. 406, L63 (2003).

[26] S. Savaglio, EAS Publ. Ser. 61, 381 (2013).

[27] E. M. Levesque, Publ. Astron. Soc. Pac. 126, 1 (2014).

[28] A. Cucchiara, M. Fumagalli, M. Rafelski et al., arXiv:1408.3578.

[29] A. S. Fruchter et al., Nature (London) 441, 463 (2006).

[30] K. M. Svensson, A. J. Levan, N. R. Tanvir, A. S. Fruchter, and L.-G. Strolger, Mon. Not. R. Astron. Soc. 405, 57 (2010).

[31] E. Nakar, Phys. Rep. 442, 166 (2007).

[32] E. Berger, arXiv:1311.2603 [Annu. Rev. Astron. Astrophys. (to be published)].

[33] D. Wanderman and T. Piran, arXiv:1405.5878.

[34] A. M. Soderberg, S. R. Kulkarni, E. Nakar et al., Nature (London) 442, 1014 (2006).

[35] L. Casagrande, R. Schönrich, M. Asplund, S. Cassisi, I. Ramírez, J. Meléndez, T. Bensby, and S. Feltzing, Astron. Astrophys. 530, A138 (2011).

[36] B. Panter, R. Jimenez, A. F. Heavens, and S. Charlot, Mon. Not. R. Astron. Soc. 391, 1117 (2008).

[37] L. M. Ejzak, A. L. Melott, M. V. Medvedev, and B. C. Thomas, Astrophys. J. 654, 373 (2007).

[38] B. Panter, R. Jimenez, A. F. Heavens, and S. Charlot, Mon. Not. R. Astron. Soc. 378, 1550 (2007).

[39] P. J. McMillan, Mon. Not. R. Astron. Soc. 414, 2446 (2011).

[40] J. Bovy and H.-W. Rix, Astrophys. J. 779, 115 (2013). 